\title{
Programmable source for DC voltage ratio calibration of DVMs
}

\author{
R. Cerri ${ }^{1}$, A.Sosso ${ }^{2}$, P. P. Capra ${ }^{3}$, L. Roncaglione ${ }^{4}$ \\ 1,2,3 National Institute of Metrological Research, strada delle Cacce 91, 10135 TURIN - Italy \\ ${ }^{1}+390113919426$ r.cerri@inrim.it \\ ${ }^{2}+390113919436$ a.sosso@inrim.it \\ 3390113919424 p.capra@inrim.it \\ +390113919418 I.roncaglione@inrim.it
}

\begin{abstract}
Résumé. Les multimètres numerale de haute qualité, en raison de leur linéarité, peuvent être utilisés comme normes de rapport de tension. Les applications possibles de métrologie sont nombreuses: les laboratoires secondaires, peuvent utiliser avec profit un multimètre numérique avec une courbe de linéarité calibrée pour la maintenance et la traçabilité metrologique de l'échelle du volt et pour l'étalonnage des rapports de résistance. Dans les laboratoires primaires, un multimètre numerale calibré avec une grande exactitude est un moyen efficace de maintenir l'échelle de résistance. La linéarité est mieux déterminée dans la gamme $\pm 10 \mathrm{~V}$, l'intervalle fondamental de fonctionnement de convertisseurs analogique-numériques à l'intérieur de la plupart des multimétres. Cet article décrit une source de tension continue de trés faible bruit electrique, basée sur un convertisseur N/A R-2R, pour générer le tension de référence necessarie pour la calibration en linéarité d'un multimétre. Un filtre specifiquement concu pour cette application, posé a la sortie di generateur, attenue le fluctuations de tension residues et stabilise le lectures du multimetre..
\end{abstract}

\section{Introduction}

Modern digital multimeters (DMM) are essential tools in both primary metrology institutes and secondary, industrial and research laboratories that need high precision electrical measurements. Currently the DMM can take measurements on at least five functions: voltage and current ac and de resistance in extended ranges. Multimeters require, to achieve the highest accuracy, a complex calibration procedure that normally should be repeated periodically. A technology known as "artifact calibration" exists in some multimeters. It consists in the possibility of using, in the calibration step, a limited number of standards. In other words, the multimeters equipped with such technology use, during the calibration process, standards and quantity-value scales built inside the instrument. With the artifact calibration, only a dc voltage reference (es. $10 \mathrm{~V}$ ) and a standard resistor (es. $10 \mathrm{k} \Omega$ ) are required to calibrate all the five measurement functions in any range. This technology, although extremely advantageous both in economic and in accuracy terms, does not solve the problem of regular calibrations that in any case must be performed periodically because of the drift of the internal reference of which the DMMs are equipped. An advantageous way in the use of high-grade DMMs for dc voltage measurements is the use of techniques based on the linearity of analog to digital converters (ADC) that

\footnotetext{
a Corresponding author: a.sosso@inrim.it
}

constitute the heart of each DMM. The linearity of the ADCs, unlike other properties of multimeters, such as the stability of the internal reference, is stable over time and may be considered as a "distinctive mark" of each DMM. It has been shown that DVMs operated in the $10 \mathrm{~V}$ range can be successfully used as voltage ratio standards for maintenance and dissemination $[1,2]$. Specifically, the method in use at INRiM to maintain and disseminate voltage ratios makes use of a set DVMs corrected for nonlinearities used as "linearity standard" to determine by direct comparison the residual deviations from linearity in a multimeter under calibration.

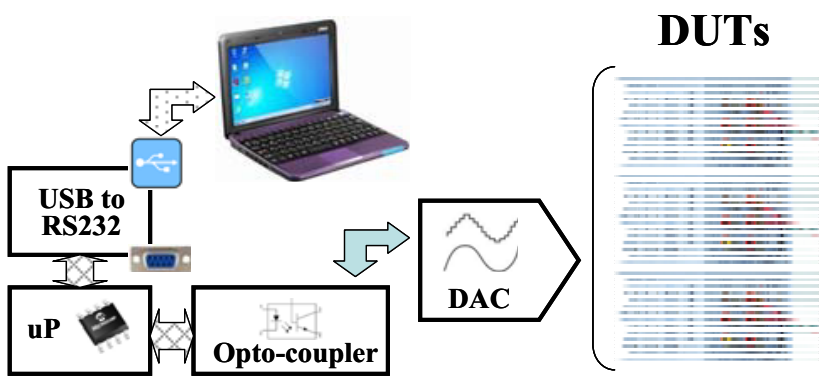

Figure 1. Block diagram of the entire system.

The paper describes a dc and low noise voltage source, developed at INRIM, specially designed to calibrate highgrade DMMs linearity. The topic of the next paragraphs 
is to show both the system developed and the techniques use at INRIM.

\section{The dc source}

The voltage generator is based on an old, low speed 16 bit digital to analog converter (DAC). The digital to analog conversion is made by means of a R-2R net, technology characterized by a low noise. The resolution of the DAC used is limited to 16 bit, but the resolution has only effect on the number of the test point available. The stability of the output voltage is enhanced by a filter that attenuates all noise contributions. Furthermore a heavy capacitor connected to the output of the voltage source provide to attenuate both the noise contribution of the $\mathrm{DAC}$ and the post-processing electronics.

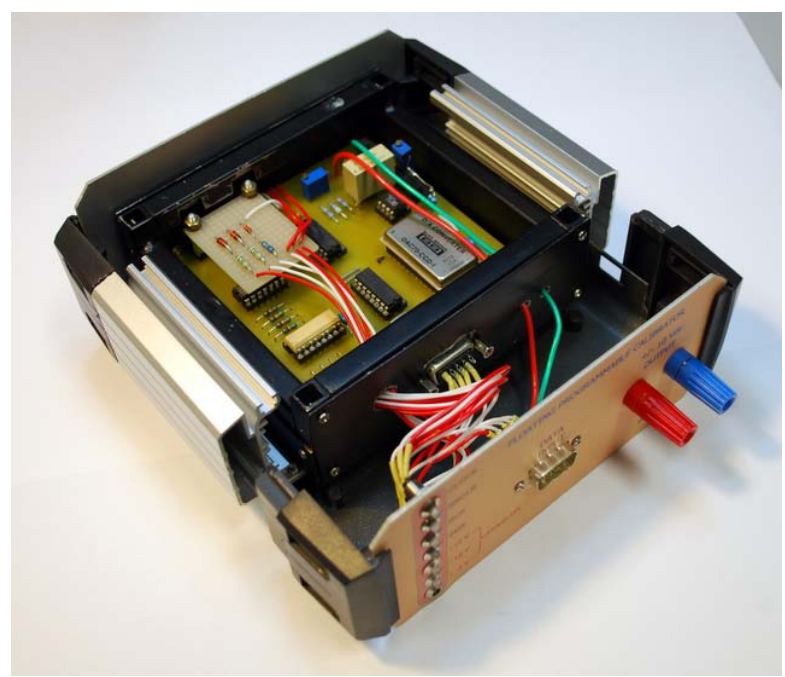

Figure 2. Photograph of the device showing the overall structure and internal circuits.

The analog parts of the system is fully battery supplied while the DAC inputs are optoisolated. The output wires that connect the source with the voltmeters under test are thus the only galvanic link to the outside environment in order to avoid ground loop

\section{DAC control and digital interface}

The DAC is controlled via the USB port of a computer to minimize the effects and disturbs products by the body capacitance of an operator [3]. For convenience is also possible to set the voltage by acting manually on the control circuit The control system consists of two circuits. The first is an embedded card based on a common 8-bit microprocessor of the family $18 \mathrm{~F}$ produced by Microchip.

The microprocessor circuit is low power and is then directly supplied from the computer. Four pins on the microprocessor (uP), configured as digital outputs, are connected to a pair of cascading shift register serial-in parallel-out. The sixteen parallel outputs of the shift register are connected to the digital inputs of the DAC placed on the second board card equipped with battery power. The inputs of the two shift registers are electrically isolated from the uP with an optocoupler which prevents the crosstalk noise generated by the computer or microprocessor to the DAC. Furthermore, since the converter operates in quasi-static conditions, i.e. the voltage remains constant during DVMs readings, the digital codes are sent to the DAC only when the DVMs are idle, avoiding any disturbance on readings via inductive or capacitive coupling with the digital lines.

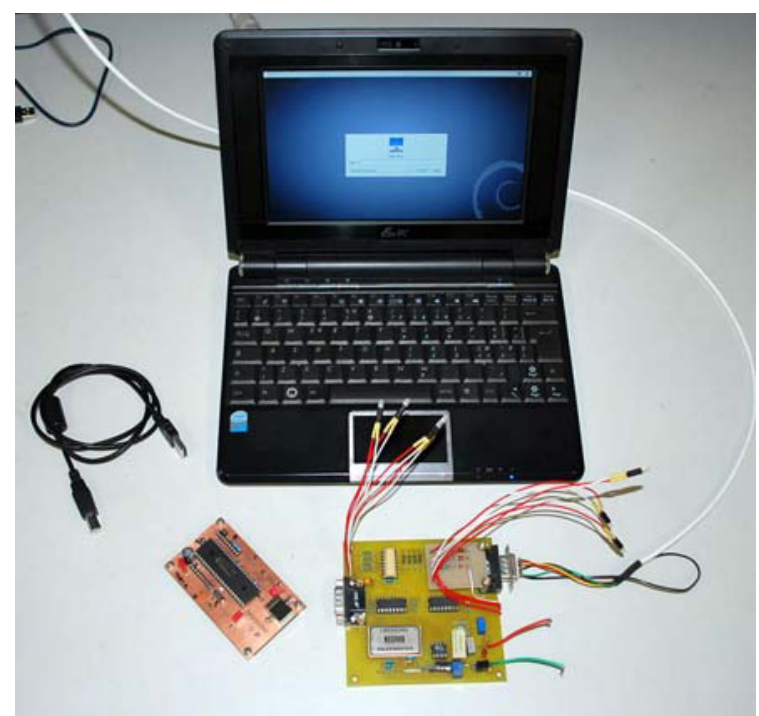

Figure 3. Caption of the Figure 1. Below the figure.

The system is controlled by means of an interface connected to a pc with a USB or a serial interface. A program developed in VB6 controls the digital multimeter under test and the DC voltage generator. The picture reports the appearance of the graphical program interface.

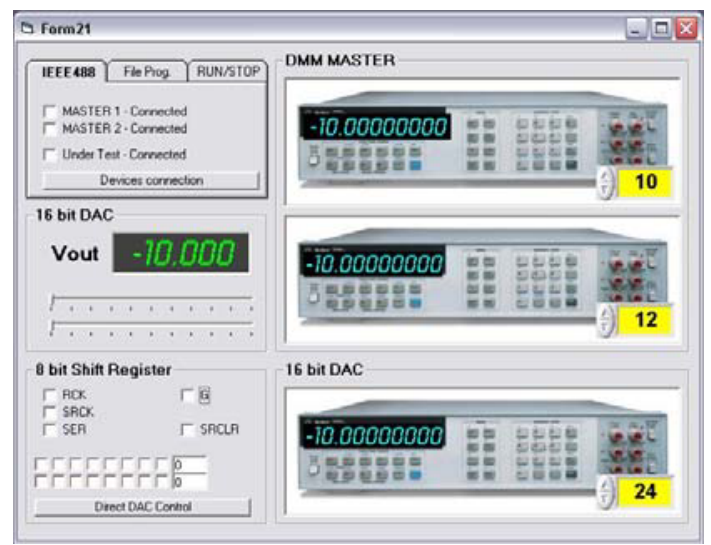

Figure 4. Graphical interface of the controlling program Developed in Visual Basic. All the calibration procedure is performed automatically. In the picture the two DMMs on the top are the references while the DMM on the top is under calibration

\section{DVM linearity calibration}

When used for a linearity transfer; from a DVM with known nonlinearities [4] the output of the standard is directly connected to one (or more) DVM under calibration so that all see the same voltage from the low noise source. The voltage applied to the DMMs is changed with suitably spaced steps to span the whole 
DVM range, recording measured values of all instruments. Provided that any relevant drift is linear and samples are read with equal time interval separation to preserve linearity vs. sample number. A linear fit of the readings of an instrument in calibration vs. the linearity standard is then performed, and finally nonlinearities are calculated as the differences between the actual readings and the corresponding value given by the fit line. Another assumption for the calculation to be correct is the exact coincidence of integration window times, which cannot be satisfied in practice. GPIB bus timing error in the synchronization of the sampling intervals via the interface device-independent trigger, can be estimated in some microseconds.

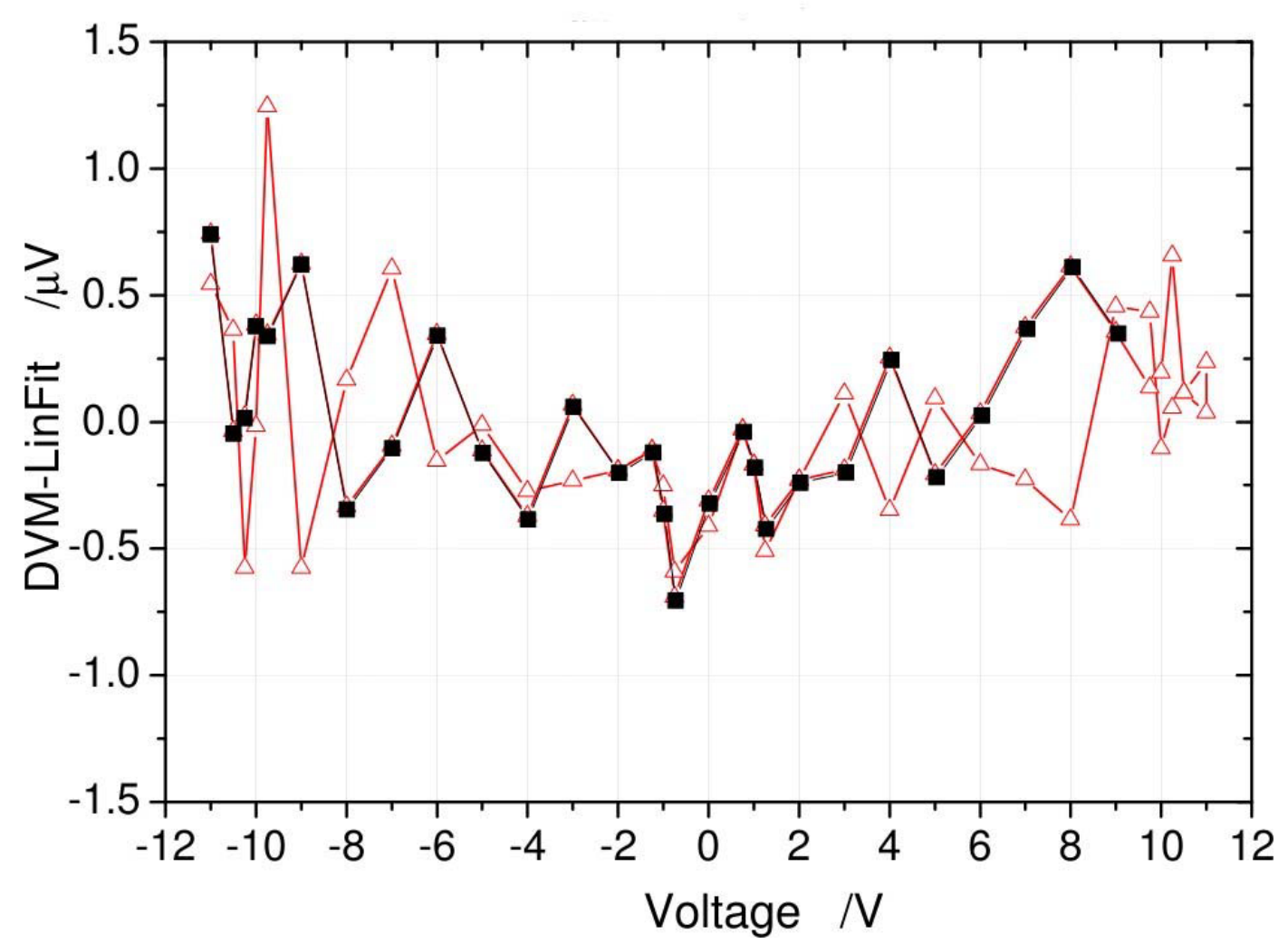

Figure 4. Nonlinearities of a high accuracy DMM measured with the presented calibration method.

The source noise sampled at different instants, and integrated over the aperture window adds a contribution to the DC readings with random fluctuations among instruments under calibration. Our source with low noise provides the most straighforward technique for reducing this uncertainty source.

A specific beneficial side effect of the output capacitor in the source is represented by the reduction of any mutual disturbance between DVMs due to pump-out currents [4], owing to the shorting effect of the capacitor at high frequencies. However, several issues are raised by the capacitive loading of the amplifiers at the DAC output, and the final stage has to be specifically designed to avoid ringing and auto-oscillations induced by the signal phase rotation from the reactive load.

A first prototype with a few $\mathrm{pF}$ capacitor was successfully tested, showing no spurious oscillations. Yet, additional work is required to define the best compromise between noise reduction through strong low pass filtering and a practical speed in transitions when switching voltage level, to reduce measurement time.

\section{DVM linearity and gain error evaluation}

The measurement procedure is straightforward: the output of the "linearity standard" is directly connected to the DVM under calibration so that both see the voltage across the same potential nodes. The voltage is varied to span the whole DVM range considered, with the low noise voltage source. A linear fit of the readings of the instrument under calibration vs. the linearity standard used is then performed. Finally the differences between the actual readings and the value given by the fit line, represent the nonlinearities (Fig. 4). The procedure also provides the actual DVM gain (gain error) and the total voltage offset. If offsets and thermal e.m.f.s are not constant but change linearly with time, their effect may result in a modified DVM gain or an added nonlinearity (if samples are not taken at instants evenly spaced in time), and must be eliminated from result. The added contribution to slope due to drift can be canceled taking a first set of readings with positive increments, then, a 
second one, backwards, with negative increments. Any relevant drift assumed to be linear; samples are read with equal time interval separation to preserve linearity vs. sample number.

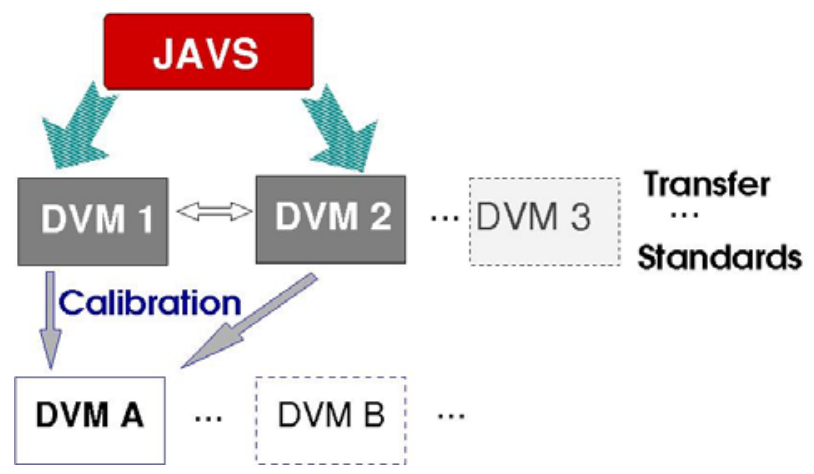

Figure 5. Paths for calibration and consistency check for calibrated DVMs. DVM1, DVM2 are independently ratiocalibrated against a JAVS and can be used to calibrate DVMA, DVMB, ... A calibrated multimeter (DVM1) can be used also for the calibration of another linearity standard (DVM2), providing a consistency check suitable for metrological confirmation.

\section{Conclusion}

A new ultra low noise voltage source suited to DC electrical metrology requirements was built and tested. Owing to its completely floating circuitry, interferences, ground loops and noise induced by unwanted electrical couplings are reduced with respect to a mains supplied source (e.g. a calibrator). The application to the determination of residual non linearity of DVMs is particularly interesting and was studied to estimate the advantages with respect to solutions based on commercial instrumentation. According to first experiments, the device is expected to reduce DVM voltage ratio uncertainty to a value close to $1 \cdot 10^{-8}$ in the full range.

\section{References}

[1] C. Cassiago, R. Cerri, G. La Paglia, and A. Sosso,: Application of DMM linearity to DC volt traceability in CPEM 2004 Conf. Dig., 2004, pp. $1761 ; 177$.

[2] G. Boella, P. Capra, C. Cassiago, R. Cerri, G. M. Reedtz, and A. Sosso, \&Traceability of the 10 k\&Omega; standard at IEN; IEEE Trans. Instr. Meas., vol. 50, no. 2, pp. 245-248, Apr. 2001.

[3] H. W Ott Wiley-Interscience publication, second edition, ISBN 0-471-85068-3, (1988).

[4] W. Goeke, R. Swerlein, S. Ventzke, and S. Stever, Calibration of an $81 / 2$ digit multimeter from only two external standards; Hewlet-Packard Journal, Apr. 1989. 